Recent Insights into the Physics of the Sun and Heliosphere:

Highlights from SOHO and Other Space Missions

IAU Symposium, Vol. 203, 2001

P. Brekke, B. Fleck, and J. B. Gurman eds.

\title{
The Relationship between CMEs and Prominence Eruptions
}

\author{
B. Schmieder and L. van Driel-Gesztelyi
}

Observatoire de Paris, 92195 Meudon, Cedex Principal, France

C. Delannée

NASA/GSFC, Code 682.3, Greenbelt, MD 20771, USA

G. M. Simnett

School of Phys. and Astro., University of Birmingham, B15 2TT, UK

J. E. Wiik

Institut of Astrophysics, University of Oslo, Blindern, Norway

\begin{abstract}
We have studied the temporal relationship between three selected prominence eruptions and their associated coronal mass ejections (CME). For these three events it is not possible to predict from the CME time history when the prominence would erupt. However, both are signatures of destabilisation of the global coronal magnetic field. The lack of a precise temporal relationship reflects the fact that several coronal stuctures are destabilised, and in general one will predominate in the CME structure. If this structure happens to be the same as that constraining the prominence, then the latter will erupt following the launch of the CME. If the prominence is constrained by a separate magnetic structure, then it may erupt before or after the main CME. Reconfiguration of the global coronal field probably drives a fast increase of the poloidal field in the prominence flux tube or introduces destabilising shear forces. Events on May 1, 1996, September 25-27, 1996, and May 311997 are discussed.
\end{abstract}

\section{MAY 311997}

A mid-latitude prominence was observed before and after its eruption with SOHO and with the Meudon and Pic du Midi observatories, as part of a MEDOC campaign in Orsay (Schmieder et al. 2000). A few hours before its eruption, the prominence is partially heated (as seen in $195 \AA$ with EIT). The physical conditions of the prominence before its eruption have been investigated by spectroscopic analysis of SUMER (the Lyman series L4 to L9) and of CDS combined with the Multi-channel Subtractive Double Pass Spectrograph (MSDP) spectra of $\mathrm{H} \alpha$. Five hours before the eruption, large broadenings of chromospheric and transition region lines (CDS) were observed in the main body of the prominence 

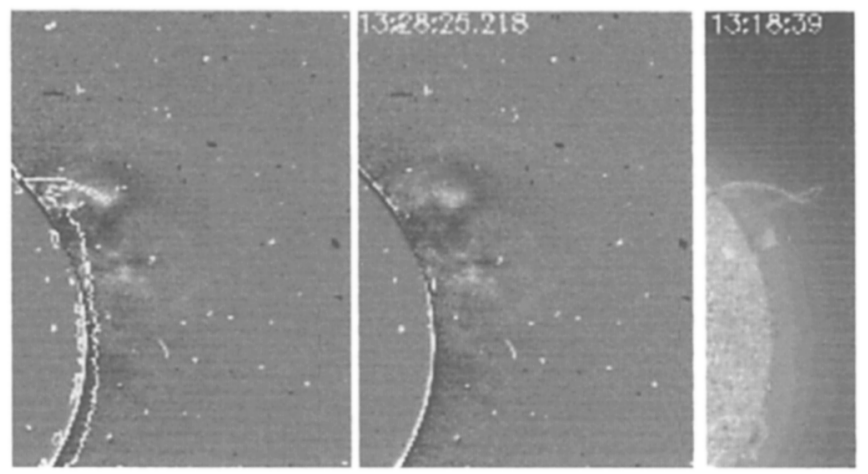

Figure 1. The May 311997 CME observation by LASCO C1 (middle panel); the prominence eruption observed by EIT $304 \AA$ (right panel); and the overlay of the $304 \AA$ EIT prominence at 13:18 UT (white contours) with the Fe XIV C1 image at 13:28 UT (left panel).

suggesting strong turbulence as well as opposite Dopplershifts on each side of the prominence ( $\mathrm{H} \alpha$ and $\mathrm{He} \mathrm{I}$ ) which could be interpreted as twisting motions of a few $\mathrm{km} / \mathrm{s}$. In that period part of the prominence was escaping, showing a high Doppler shift as derived from the Lyman line profiles. An X-ray bright point observed close to a prominence footpoint could be the signature of reconnection process linked to the destabilization of the prominence.

At 13:18 UT EIT (304 A) observed the eruption of the prominence $(\sim 100$ $\mathrm{km} / \mathrm{s}$ ). The CME was first detected by LASCO C1 with three components: a bright leading loop with a velocity of around $9 \mathrm{~km} / \mathrm{s}$ up to 13:28 UT, a cavity and the prominence (Fig. 1). We notice that both, the prominence and the bright loop, are deviated towards the equator. This implies that they belong to the same global expansion event constrained to remain in the equatorial streamer.

There is a gap in the observations made by LASCO. When LASCO is back around 18:30 UT, the CME in C3 was accelerating, and was quite slow (less than $100 \mathrm{~km} / \mathrm{s}$ ) below $4 \mathrm{R}_{\odot}$ solar radii, but by $9 \mathrm{R}_{\odot}$ it is $350 \mathrm{~km} / \mathrm{s}$.

\section{May $1 \mathbf{1 9 9 6}$}

Observations of an eruptive prominence were obtained on May 1, 1996, between 07:13-11:00 UT, with the SUMER and CDS instruments (Wiik et al. 1997). The prominence was huge, located between S20 and S40 on the east limb reaching an altitude of $100,000 \mathrm{~km}$ at the time of the observations. A coronal mass ejection observed with LASCO C3 is associated temporally and spatially with this prominence. The CME had significant structure, and we can follow the leading edge versus time and extrapolate back to the onset. The CME appeared to have started prior to the prominence eruption (Fig. 2). The main important characteristics are: the presence of the prominence channel outlined by bright Yohkoh 

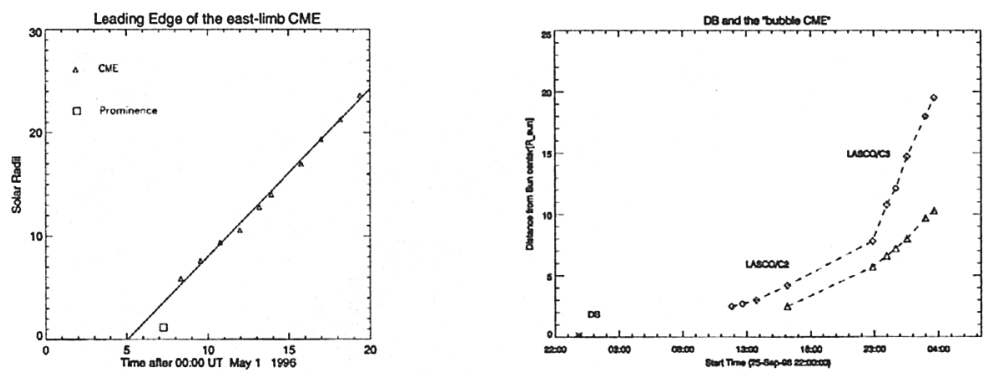

Figure 2. Time diagrams (a) May 11996 evolution of the leading edge of the CME observed by LASCO C3 coronograph. The top of the prominence observed by SUMER at 07:25 UT is indicated by the square, (b) Sept 251996 evolution of the leading edge (losange) and the following edge (triangle) of the CME observed by $\mathrm{C} 2$ and $\mathrm{C} 3$, the time of the eruption of the prominence is indicated by $\mathrm{DB}$

soft X-rays, the twisted prominence detected by the SUMER Dopplershifts. The $\mathrm{CME}$ is offset equatorward from the associated prominence.

\section{September 251996}

The CME (Fig. 3) was related to a disparition brusque of a prominence (DB) in the dispersed bipolar remnant of the active region NOAA 7986 (4th rotation of the AR). The long prominence lay along the magnetic inversion line reaching as far South as the polar crown, where the inversion line turned westward forming a "switchback". During the hours prior to the DB along the prominence channel at different locations flux emergence and cancellation took place accompanied by coronal activity. Prior to the eruption the prominence showed turbulent motions for more than an hour and it gradually disappeared in the He $10830 \mathrm{~A}$ line by 23:45 UT on September 25. A slow swelling of the AR loops was seen in EIT and LASCO C1 well before the DB.

The DB only made the prominence south of the AR disappear, while the prominence inside the AR was only heated up, appearing in emission in three EIT wavelengths (in the Fe lines) and in SXRs (as observed between 00:2401:46 UT). In SXRs cusped arcade formation started immediately after the DB (23:46 UT) followed by an arcade formation along the polar-crown part of the switchback inversion zone (Fig. 3). The LASCO images of September 25-27 1996 showed a CME spanning several days. The "primary" CME appeared in C3 as a "ragged front" between 23:25 UT on 25 Sept. and 01:25 UT. On September 26, LASCO C2 detects streamer brightening in the W-SW at 06:35 UT and a "bubble" became visible in the C2 field-of-view at about 9:12 UT. The CME spanned almost $140^{\circ}$ in position angle (PA) and was centered at $\mathrm{PA}=205^{\circ}$. There were several dark and bright loops embedded in the ragged front and the height-time measurements for an early feature indicate a gradual acceleration through the C2 with a velocity reaching $100 \mathrm{~km} / \mathrm{s}$ around $10 \mathrm{R}_{\odot}$ and C3 field with a final speed of about $500 \mathrm{kms}^{-1}$ at $29 \mathrm{R}_{\odot}$ on September 27 (Fig.2). It 


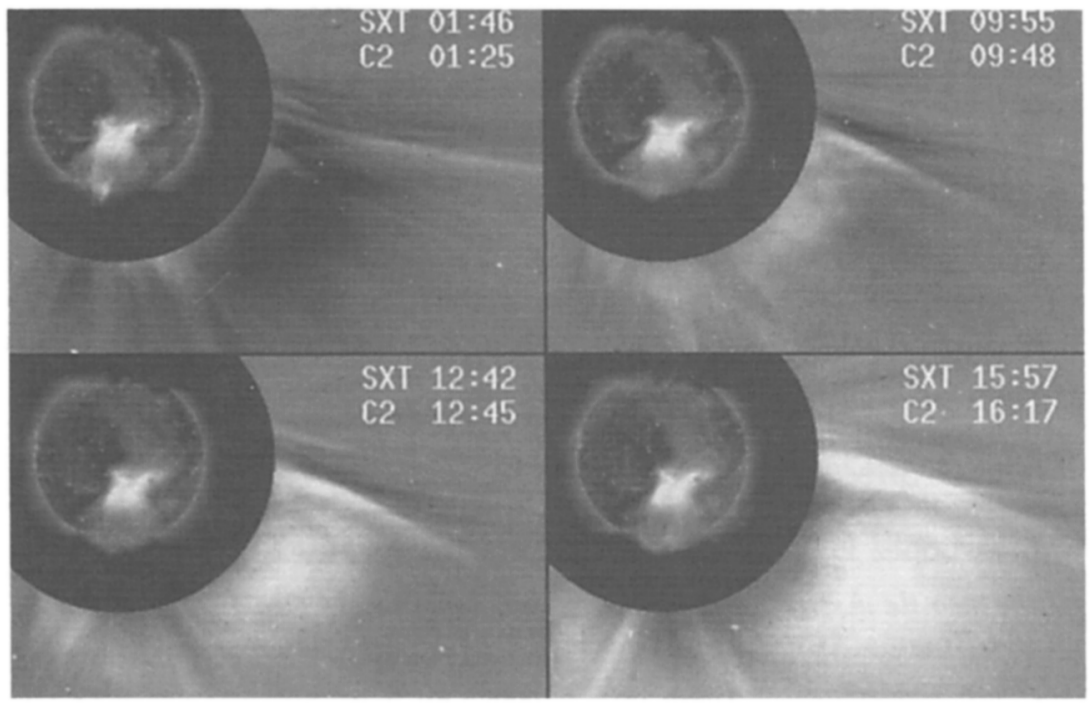

Figure 3. Co-aligned Yohkoh/SXT and SOHO/LASCO C2 images show the evolution of the lower coronal structures and the CME on September 261996

is not clear if we are observing the same structure. It is possible in this very complicated event, which is covering a large part of the polar crown, has more than one CME associated with the destabilisation, may be three.

\section{Conclusion}

The filament filament can be interpreted in the framework of new MHD modeling of lateral prominence footpoints and flux rope (Aulanier and Démoulin 1998). The observations of CMEs are consistent with a model in which the main part of the CME is within the equatorial streamer belt, and that it removes, or weakens, the coronal magnetic field at higher latitudes, thereby affecting any underlying structures at these latitudes which have been relying on the coronal field for their stability. Prominence eruptions and CMEs seem to be an ensemble of elementary events not necessarily directly related to each other but belonging to the same global instability of the magnetic structure, possibly being consequences of each other.

\section{References}

Aulanier, G. and Démoulin, P.: 1998, A\&A; 329, 125

Schmieder B., Delannée, C., Deng Y. et al.: 2000, A\&A358, 728

Wiik, J., Schmieder, B., Kucera, T., Simnett, G.: 1997, Solar Phys.,175, 411 